\title{
Rendimento de soja em sistema de integração lavoura-pecuária: efeito de métodos e intensidades de pastejo
}

\author{
Soybean yield in an animal-crop rotation system: effects of grazing methods and intensities
}

\author{
Robson Lunardi ${ }^{*}$ Paulo César de Faccio Carvalho" Carlos Ricardo Trein ${ }^{\text {II }}$ José Antonio Costa ${ }^{\text {II }}$ \\ Guilherme Fernandes Cauduro"I Cristina Maria Pacheco Barbosa" \\ Angelo Antônio Queirolo Aguinaga ${ }^{I I}$
}

\section{RESUMO}

Sistemas de integração lavoura-pecuária têm despertado o interesse de produtores que buscam a diversificação das atividades $e$ o aumento da rentabilidade. $O$ objetivo deste trabalho foi avaliar a influência de métodos e intensidades de pastejo de ovinos no rendimento da soja cultivada em dois espaçamentos entre linhas, em um sistema de integração lavoura-pecuária. O experimento foi conduzido no município de Eldorado do Sul - RS, na Estação Experimental Agronômica - UFRGS na safra 2003/2004, cujas coordenadas geográficas são $30^{\circ} 05^{\prime} 22^{\prime}$ ' $S$ de latitude e 51039'08” W de longitude. O delineamento experimental foi em blocos casualizados num fatorial com duas intensidades de pastejo, baixa e moderada, dois métodos de pastejo, contínuo e rotacionado, e dois espaçamentos entre fileiras de soja, 0,2 e $0,4 \mathrm{~m}$, com quatro repetições. Uma área foi adicionada como testemunha sem pastejo. Avaliaram-se o rendimento e os componentes do rendimento da soja. A produção de soja foi superior nos tratamentos submetidos a pastejo em comparação aos não-pastejados. Dentre os pastejados, o rendimento de soja foi superior na intensidade de pastejo baixa em comparação com a intensidade moderada. A intensidade de pastejo utilizada no inverno é o principal determinante do sucesso desse sistema de integração lavoura-pecuária.

Palavras-chave: Lolium multiflorum, Glycine max, lotação contínua, lotação rotacionada, rendimento de soja em sistema de integração lavourapecuária: efeito de métodos e intensidades de pastejo.

\section{ABSTRACT}

Animal-crop rotation systems are fostering the interest of farmers searching for diversification and the increase of profitability. The experiment aimed to evaluate the influence of grazing methods and intensities applied on winter pastures grazed by lambs and its consequence for soybean yield cultivated in succession. This crop-animal rotation trial was conducted in 2003/2004 at UFRGS Agricultural Research Station (Universidade Federal do Rio Grande do Sul) in Eldorado do Sul, Rio Grande do Sul state, Brazil, whose geographical coordinates are $30^{\circ} 05^{\prime} 22$ " S latitude and 51039'08" W longitude. The experimental design was a randomized complete block arranged in a factorial with two grazing methods, continuous and rotational, two grazing intensities, low and moderate, and two soybean rows spacing, 0.2 and $0.4 \mathrm{~m}$, with four replicates. An additional area was surveyed as a no grazing paddock reference and soybean yield attributes were evaluated. Soybean yield was superior in grazed areas $(P<0.05)$ compared to non grazed ones. Among grazed treatments, soybean yield was superior $(P<0.05)$ at low grazing intensity compared to moderate grazing intensity. The grazing intensity level used during winter is fundamental to the success of animal-crop rotation systems.

Key words: Lolium multiflorum, Glycine max, continuous grazing, rotational grazing, soybean yield in an animal-crop rotation system: effects of grazing methods and intensities.

\section{INTRODUÇÃO}

A adoção do sistema de integração lavourapecuária pelos produtores gera, em muitos casos, dúvidas quanto à conveniência desta tecnologia. Isso ocorre, principalmente, quando o solo é cultivado em sistema de plantio direto ou quando, devido ao manejo adotado, o solo fica exposto ao ambiente. Como conseqüência, freqüentemente convive-se com o paradigma quanto ao alegado impacto negativo dos

\footnotetext{
IDepartamento de Plantas Forrageiras, Universidade Federal do Rio Grande do Sul (UFRGS), Porto Alegre, RS, Brasil. E-mail: robson.lunardi@syngenta.com.*Autor para correspondência.
}

IIUFRG, Porto Alegre, RS, Brasil. 
animais no rendimento das culturas em sucessão (CARVALHO et al., 2005). O que ocorre, na realidade, são apenas estimações visuais de um suposto impacto do pisoteio animal que, não se comprovam com os dados gerados pelas pesquisas em diversas citações bibliográficas (MORAES \& LUSTOSA, 1997). Com isso, limita-se a adoção de uma tecnologia que se mostra fundamental na diversificação e na rotação dos sistemas agrícolas, na estabilidade e no incremento de rentabilidade às explorações agrícolas (CARVALHO et al., 2005).

É provável que esse paradigma seja uma herança de épocas em que predominava o uso de agricultura convencional, em que utilizava-se o preparo do solo, aliado ao uso incorreto de lotações excessivas na rotação com a pecuária (MORAES \& LUSTOSA, 1997). Quando adequadamente manejada, a integração pode, inclusive, potencializar a produção da lavoura (ASSMANN, 2001). FONTANELI et al. (2000) concluíram que a introdução de pecuária em áreas agrícolas não foi negativa quando as pastagens foram adequadamente manejadas.

A principal variável definidora do sucesso ou insucesso de sistemas integrados é a taxa de lotação empregada, por seus efeitos diretos e indiretos sobre a quantidade de forragem e de nutrientes que ciclam no sistema (CARVALHO et al., 2005). Em lotações elevadas, o pisoteio animal pode promover impactos negativos em atributos físicos do solo (TREIN et al., 1991; MORAES \& LUSTOSA, 1997). Ainda que esse impacto negativo seja, na maioria das vezes, superficial (MORAES \& LUSTOSA, 1997), a intensidade de pastejo deve ser conduzida com muito critério.

Com relação ao efeito dos métodos de pastejo sobre o rendimento das lavouras em sucessão, nos sistemas de integração lavoura-pecuária, constatase uma quase inexistência de registros na literatura. $\mathrm{O}$ método de pastejo escolhido para manejo da pastagem, no ciclo da pecuária, pode ser tão importante quanto a intensidade de pastejo definida. Enquanto o método de pastejo em lotação contínua permite que os animais tenham distribuição aleatória em toda a área, a lotação rotacionada se caracteriza pelo controle maior do pastejo e por elevadas densidades instantâneas de animais por unidade de área.

Um outro fator potencialmente importante em sistemas integrados é o espaçamento entre fileiras da lavoura em sucessão. A redução do espaçamento entre fileiras de soja de $0,4 \mathrm{~m}$ para $0,2 \mathrm{~m}$ pode propiciar melhor arranjo espacial com diminuição da competição intra-específica (VENTIMIGLIA et al., 1999), aumentando o potencial de rendimento da cultura.
A fim de obter subsídios para o manejo do animal em áreas de integração lavoura-pecuária, objetivou-se estudar um sistema de integração, em que a produção de soja e a terminação de cordeiros foram associadas. Avaliou-se a influência do manejo da pastagem, em particular do método e da intensidade de pastejo, e do espaçamento entre linhas, no rendimento da cultura da soja em sucessão.

\section{MATERIAL E MÉTODOS}

O experimento foi conduzido na Estação Experimental Agronômica da Universidade Federal do Rio Grande do Sul (EEA/UFRGS), no município de Eldorado do Sul - RS, em Argissolo Vermelho Distrófico típico (EMBRAPA, 1999), levemente ondulado, sem limitações para cultivos. As coordenadas geográficas da EEA/UFRGS são 30 05’22” S de latitude e 51'39'08” $\mathrm{W}$ de longitude. O clima da região é subtropical úmido com verões quentes, tipo fundamental "Cfa" da classificação climática de Köppen.

A semeadura da pastagem de azevém foi realizada em 21/04/2003, com semeadora-adubadora de plantio direto, com espaçamento entre fileiras de $0,17 \mathrm{~m}$, depois da dessecação da área experimental com $5 \mathrm{~L} \mathrm{ha}^{-1}$ do produto comercial (p.c.) do herbicida de ingrediente ativo (i.a.) glyphosate. A quantidade de semente utilizada foi de $32 \mathrm{~kg} \mathrm{ha}^{-1}$ de azevém (Lolium multiflorum Lam) cultivar "Comum RS” (MEDEIROS \& NABINGER, 2001).

As amostras de solo foram coletadas em março de 2003 na profundidade de $0-0,1 \mathrm{~m}$. Os resultados da análise de solo revelaram: argila (\%), 19,0; pH ( $\left.\mathrm{H}_{2} \mathrm{O}\right)$, 5,3; M.O. (\%), 2,9; P (mg L $\left.{ }^{-1}\right), 13,5 ; \mathrm{K}\left(\mathrm{mg} \mathrm{L}^{-1}\right)$ 135,0; Al trocável $\left(\mathrm{cmol}_{\mathrm{C}} \mathrm{L}^{-1}\right), 0,3$; Ca trocável $\left(\mathrm{cmol}_{\mathrm{C}} \mathrm{L}^{-1}\right)$, 2,1; $\mathrm{Mg}\left(\mathrm{cmol} \mathrm{L}^{-1}\right), 1,2$; saturação de bases da CTC (\%), 54,2. A adubação e a calagem utilizadas seguiram a Recomendação de Adubação e Calagem para os Estados do RS e SC (COMISSÃO DE FERTILIDADE DO SOLO-RS/SC, 1995). Aplicou-se 1 tha $^{-1}$ de calcário faixa C (em 16/4/2003) e 200 $\mathrm{kg} \mathrm{ha}^{-1}$ de adubo $\mathrm{N}-\mathrm{P}_{2} \mathrm{O}_{5}-$ $\mathrm{K}_{2} \mathrm{O}$ na fórmula 5-20-20 em (21/4/2003). Também foram utilizados $150 \mathrm{~kg}$ de $\mathrm{N} \mathrm{ha}^{-1}$ sob forma de uréia em duas coberturas: metade da dose aplicada na emissão da $4^{\mathrm{a}}$ folha de azevém e o restante no início do período de primavera. Aárea total utilizada foi de aproximadamente 6ha. Desse total, 1,7ha foram destinados aos animais reguladores e 4,3ha constituíram as unidades experimentais.

Os tratamentos consistiram de duas intensidades de pastejo (moderada - IPM e baixa - IPB), definidas por ofertas de forragem, representando, respectivamente, 2,5 e 5,0 vezes o potencial de consumo 
dos animais, e dois métodos de pastejo (lotação contínua - LC e lotação rotacionada - LR). Foi introduzida parcela subdividida com dois espaçamentos entre fileiras na soja de 20 e $40 \mathrm{~cm}$. Uma área sem pastejo foi utilizada como tratamento testemunha.

O tempo de duração de vida da folha (TVF) do azevém foi utilizado para determinação dos ciclos de pastejo. Utilizou-se informações de experimentos na EEA/UFRGS, em que foi mensurado o TVF de $500^{\circ} \mathrm{C}$ folha ${ }^{-1}$ no período de junho a agosto e $410^{\circ} \mathrm{C}_{\text {folha }}{ }^{-1} \mathrm{de}$ setembro a novembro (PONTES et al., 2003 e FREITAS, 2003). Dividiu-se o TVF pela média de temperatura dos meses de junho a novembro, caracterizando então quatro ciclos de pastejo em que a oferta de forragem era ajustada (período I: 12/7 a 15/8; II: 16/8 a 16/9; III: 17/9 a 9/10; IV: 10/10 a 31/10). O período de ocupação foi de dois dias. Para a determinação dos subpotreiros do método LR, dividiu-se o comprimento das unidades experimentais (UE) pelo número de dias do ciclo de pastejo.

Em ambos os métodos, foi utilizada lotação variável por meio de animais reguladores, segundo a técnica “put-and-take” (MOTT \& LUCAS, 1952). Utilizaram-se cordeiros machos inteiros, com idade média inicial de nove meses, provenientes de cruzamento entre as raças Texel e Ile de France. Para correta comparação entre métodos de pastejo, os períodos de ajuste da oferta obedeceram a TVF, ou seja, a duração do ciclo de pastejo definida na LR. O período de utilização da pastagem foi de 12/07/2003 a 02/11/2003.

Em 15/11/2003, procedeu-se aplicação de herbicida de ingrediente ativo (i.a.) Glyphosate na dosagem de $5 \mathrm{~L} \mathrm{ha}^{-1}$ do produto comercial (p.c.), e, em 26/11//2003, semeou-se a cultivar de soja "BRS 154" inoculada, com semeadora-adubadora de plantio direto. Neste momento, dois outros tratamentos foram sobrepostos sobre aqueles conduzidos no inverno, correspondendo a dois espaçamentos de plantio. As densidades de sementes foram de 7 sementes $\mathrm{m}^{-1} \mathrm{e}$ de 14 sementes $\mathrm{m}^{-1}$ para os espaçamentos entre linhas de $0,2 \mathrm{~m}$ e $0,4 \mathrm{~m}$, respectivamente. Ambos objetivaram a densidade de 350.000 sementes $\mathrm{ha}^{-1}$. A adubação no sulco de semeadura foi de $400 \mathrm{~kg} \mathrm{ha}^{-1}$ de adubo $\mathrm{N}-\mathrm{P}_{2} \mathrm{O}_{5}-$ $\mathrm{K}_{2} \mathrm{O}$, da fórmula 5-20-20.

Os tratos culturais foram: aplicação de herbicida de i.a. Imazethapyr $1,5 \mathrm{~L} \mathrm{ha}^{-1}$ de p.c. em 23/12/ 2003; aplicação de 0,5L ha-1 de herbicida de i.a. Tethraloxydin em 24/12/2003; aplicação de mistura de tanque com herbicida de i.a. Tethraloxydin 0,63L ha-1 + Imazethapyr $1 \mathrm{~L} \mathrm{ha}^{-1}+$ óleo Dash $0,5 \mathrm{~L} \mathrm{ha}^{-1}$. Foi realizada uma aplicação de inseticida de i.a. Clorpirifós com 0,5L ha ${ }^{-1}$ de p.c.; e uma aplicação de inseticida de i.a. Metamidafós com $0,8 \mathrm{~L} \mathrm{ha}^{-1}$ de p.c.

Estudou-se a presença de plantas daninhas por meio de amostra destrutiva realizada em 19/12/2003. Após a contagem do número de plantas daninhas, cortou-se quadrados de 50 x $50 \mathrm{~cm}$, coletando-se cinco amostras por UE, que foram secas em estufa de circulação de ar forçada a $65^{\circ} \mathrm{C}$ durante $48 \mathrm{~h}$ e pesadas, originando a variável massa de plantas daninhas ha-1 .

Em 10/2/2004, coletou-se amostras para avaliação do rendimento da soja, quatro amostras de $4,8 \mathrm{~m}^{2}$ por UE. Nestes mesmos pontos de amostragem, estimou-se a população de plantas por hectare. As amostras foram trilhadas, limpas e secas para obtenção do rendimento de grãos corrigidos para 13\% de umidade. Os componentes do rendimento de soja: legumes por planta, grãos por legume e peso de 1.000 grãos, foram avaliados por meio de amostras destrutivas em 19/5/2004, cinco amostras de $1 \mathrm{~m}$ de fileira em cada UE, procurando-se amostrar a média de população encontrada nas amostragens anteriores.

O delineamento experimental, para os tratamentos em arranjo fatorial, foi em blocos casualizados, com quatro repetições. Os dados coletados foram analisados utilizando-se o pacote estatístico SAS versão 8.02 (SAS INSTITUTE, 2001). Quando a diferença foi significativa $(\mathrm{P}<0,05)$ entre tratamentos, a comparação de médias foi feita pelo teste DMS.

\section{RESULTADOS E DISCUSSÃO}

Para contextualizar a discussão, é necessário se destacar resultados obtidos no período de inverno, em razão de que os efeitos das formas de condução da pastagem de azevém (duas intensidades e dois métodos de pastejo), tornaram-se o substrato para implantação da soja.

A oferta real de forragem (ORF) não diferiu entre os métodos de pastejo, havendo diferença entre as intensidades de pastejo. Além disso, não houve interação entre métodos e intensidades de pastejo, o que era condição básica para a correta comparação entre os tratamentos propostos durante o período de inverno. Sendo assim, os valores de ORF para as intensidades de pastejo baixa (IPB) e moderada (IPM) foram, respectivamente, de $18,3 \%$ e $9,7 \%$ do peso vivo (PV).

Ainda como caracterização do período de inverno, destaca-se que a área pastejada apresentou menor massa residual de forragem quando comparada à área sem pastejo e que as intensidades de pastejo resultaram em massas residuais diferentes sobre o solo 
no momento do plantio da soja. Dessa forma, a massa residual foi de $798 \mathrm{~kg}$ e $3.084 \mathrm{~kg}$ de $\mathrm{MS} \mathrm{ha}^{-1}$, para a intensidade de pastejo moderada e baixa, respectivamente. A massa de forragem na situação sem pastejo foi de $8.300 \mathrm{~kg}$ de MS ha-1 como conseqüência da ausência de pastejo pelos animais. Não houve efeito para método de pastejo.

Nas áreas em pastejo, os tratamentos distintos de intensidade promoveram massas residuais diferentes, como reflexo das taxas de lotação empregadas, pois a relação entre a massa de forragem e a taxa de lotação é inversa (CASSOL, 2003; PONTES et al., 2004).

Na tabela 1, encontram-se os resultados da produção de soja. É importante ressaltar que os baixos rendimentos de soja foram conseqüência da restrição hídrica ocorrida no período de 20/2/2004 a 10/4/2004. A chuva acumulada nesse período foi de $66 \mathrm{~mm}$, coincidindo com o período de floração e enchimento de grãos. Foram encontradas diferenças na produção de soja quando comparados os rendimentos da área pastejada x testemunha (área não-pastejada) e os rendimentos entre os tratamentos provenientes das intensidades de pastejo.

O rendimento médio de grãos da área pastejada foi de $1.384 \mathrm{~kg} \mathrm{ha}^{-1}$, e a testemunha, área sem pastejo, foi de $934 \mathrm{~kg} \mathrm{ha}^{-1}$, havendo diferenças significativas. $\mathrm{O}$ acúmulo de massa de forragem no tratamento sem pastejo, possivelmente tenha imobilizado maior quantidade de nutrientes do que aquela imobilizada nas áreas pastejadas, devido à transformação da forragem em urina e fezes, acelerando o processo de ciclagem de nutrientes no sistema. $\mathrm{O}$ crescimento inicial da soja nas áreas pastejadas foi superior quando comparada a área não-pastejada, na qual a soja apresentou sintomas de amarelecimento das folhas por um período de aproximadamente 20 a 25

Tabela 1 - Produção de grãos de soja $\left(\mathrm{kg} \mathrm{ha}^{-1}\right)$ nas intensidades de pastejo em sistema de integração lavoura-pecuária. Estação Experimental Agronômica - UFRGS, 2003/2004 ${ }^{(1)}$.

\begin{tabular}{|c|c|c|c|}
\hline \multirow{2}{*}{ Atributo avaliado } & \multicolumn{3}{|c|}{ Intensidades de pastejo } \\
\hline & Moderada & Baixa & Sem pastejo \\
\hline $\begin{array}{l}\text { Produção de soja } \\
\left(\mathrm{kg} \mathrm{ha}^{-1}\right)\end{array}$ & $1.208 \pm 285 b$ & $1.559 \pm 394 \mathrm{a}$ & $\ldots$ \\
\hline $\begin{array}{l}\text { Produção de soja } \\
\left(\mathrm{kg} \mathrm{ha}^{-1}\right)\end{array}$ & \multicolumn{2}{|c|}{$1.384 \pm 339 \mathrm{a}$} & $934 \pm 375 b$ \\
\hline
\end{tabular}

Coeficiente de variação $(\mathrm{CV})=22,55 \%$.

${ }^{(1)}$ Médias, com respectivo erro padrão, seguidas de letras iguais na linha não diferem significativamente pelo teste DMS a 5\% de significância. dias, período este em que a simbiose com as bactérias Bradirhizobium não são efetivas. ASSMANN (2001) avaliou a produtividade do milho, cultivado em seqüência a pastagem consorciada de aveia, azevém e trevo branco, com doses de $\mathrm{N}$ utilizadas com lotação contínua ou sem pastejo. Esse autor verificou que as áreas pastejadas produziram mais que as áreas não pastejadas, pois a disponibilidade de $\mathrm{N}$, expressa em $\mathrm{N}^{-\mathrm{NO}_{3}}$ (mg kg${ }^{-1}$ de solo), era maior em áreas com pastejo. A transferência de $\mathrm{N}$ da pastagem via urina e fezes dos animais para a cultura sucessora diminuiu os efeitos de imobilização do nutriente na palha, favorecendo a reciclagem do $\mathrm{N}$ no sistema.

A intensidade de pastejo afetou significativamente o rendimento de soja. A intensidade moderada resultou em rendimento de $1.208 \mathrm{~kg} \mathrm{ha}^{-1}$, inferior aos $1.559 \mathrm{~kg} \mathrm{ha}^{-1}$ obtidos na intensidade baixa (Tabela 1). As diferenças encontradas devem ter origem na maior taxa de lotação dos tratamentos de intensidade moderada, em que a massa de forragem era menor. A maior lotação e seu efeito por meio da manutenção de um IAF baixo resulta em menor produção de biomassa, tanto da parte aérea, quanto das raízes, e pode limitar a absorção de nutrientes, a infiltração, as trocas gasosas e o desenvolvimento das raízes (BICKI \& SIEMENS, 1991), refletindo-se sobre a parte aérea e o rendimento de grãos (CANARACHE et al., 1984). Muito embora pudesse ser sugerido que o impacto das lotações mais elevadas no rendimento da soja pudesse ser ocasionado por respostas associadas ao efeito negativo dos animais nas características físicas do solo, comentado por MORAES \& LUSTOSA (1997), TREIN et al (1991) e BERTOL et al. (2000), o acompanhamento da densidade do solo e da macroporosidade neste experimento revelou não haver efeito consistente da intensidade de pastejo sobre os atributos físicos do solo. Foram verificadas diferenças significativas na resistência do solo à penetração em função das intensidades de pastejo, na ordem de 1,8MPa e 1,6MPa, para as intensidades de pastejo moderada e baixa, respectivamente, avaliada logo após a saída dos animais da pastagem. Isso pode ter favorecido o crescimento radicular da cultura da soja subseqüente e aumentado os índices de infiltração de água no solo.

A densidade populacional de plantas de soja foi maior no espaçamento de $0,2 \mathrm{~m}$, com 167.659 plantas ha-1 $^{-1}$ (Tabela 2). Também foi observada uma maior população de plantas para o método de lotação rotacionada (169.629 plantas ha-1) em relação à lotação contínua (155.892 plantas ha-1). No entanto, análises de regressão demonstraram a não-significância entre o rendimento e a população de plantas, dentro de cada fator principal. A baixa densidade das plantas de soja em relação ao número de sementes semeadas por 
Tabela 2 - População de soja (plantas ha ${ }^{-1}$ ) nos espaçamentos de 0,2 e 0,4 m entre fileiras em semeadura direta, em sistema de integração lavoura-pecuária. Estação Experimental Agronômica - UFRGS, 2003/2004 ${ }^{(1)}$.

\begin{tabular}{lcccc}
\hline & & & & $0,4 \mathrm{~m}$ \\
Atributo avaliado & Com pastejo & Sem pastejo & Com pastejo & Sem pastejo \\
\cline { 2 - 5 } & $181.021 \pm 14.829$ & $154.297 \pm 7.670$ & $144.499 \pm 22.379$ & $150.130 \pm 17.479$ \\
Plantas ha ${ }^{-1}$ & $167.659 \pm 11.249 \mathrm{a}$ & $147.315 \pm 19.929 \mathrm{~b}$ \\
\hline
\end{tabular}

Coeficiente de variação $(C V)=11,50 \%$.

${ }^{(1)}$ Médias, com respectivo erro padrão, seguidas de letras iguais na coluna não diferem significativamente pelo teste DMS a 5\% de significância.

hectare ocorreu devido à estiagem de aproximadamente 10 dias após a semeadura direta.

A produtividade de soja é resultante da combinação dos componentes de rendimento, número médio de plantas por área, legumes por área, grãos por legume e peso médio dos grãos. Dentre os componentes do rendimento, legumes por planta é o de maior importância (THOMAS et al., 1998). A tabela 3 mostra pelos valores que o número de legumes de soja por planta foi diferente entre a testemunha, os métodos e as intensidades de pastejo. O número de legumes por planta foi maior (45) para a área pastejada em relação à não-pastejada (28), assim como observou-se 51 e 39 legumes por planta para as intensidades de pastejo baixa e moderada, respectivamente. Dessa forma, os tratamentos que apresentaram maior número de legumes por planta apresentaram também maiores rendimentos de grãos (Tabela 1$)$.

Também foi observado, para o espaçamento de $0,2 \mathrm{~m}$, maior número de vagens por planta, que refletiu em rendimento 9\% superior em relação aos tratamentos conduzidos sob espaçamento de $0,4 \mathrm{~m}$ entre fileiras. Essa diferença no rendimento não foi diferente estatisticamente, mas indica o mesmo resultado já alcançado por outros pesquisadores, demonstrando que a redução do espaçamento entre fileiras, mantendo a mesma população, aumentou o rendimento de grãos, resultado da melhor distribuição espacial das plantas na área (VENTIMIGLIA et al., 1999).

O peso de 1.000 grãos foi maior para a área pastejada no inverno, apresentando valores da ordem de 136,1g (Tabela 3). Sendo assim, a combinação dos fatores de maior número de legumes por planta e maior peso de grãos para os tratamentos com pastejo determinaram rendimento $32,5 \%$ superior.

Quanto à avaliação de plantas daninhas, foi observado que não houve diferenças no número de plantas $\mathrm{m}^{-2}$, entre as intensidades e os métodos de pastejo e entre os espaçamentos entre fileiras de soja. A matéria seca de plantas daninhas $\mathrm{m}^{-2}$, aumentou de 2,19 para 7,20 $\mathrm{g} \mathrm{m}^{-2}$ (Tabela 4) entre a intensidade de

Tabela 3 - Legumes por planta e peso de 1000 grãos (g) de soja nos métodos e nas intensidades de pastejo em sistema de integração lavourapecuária. Estação Experimental Agronômica - UFRGS, 2003/2004 ${ }^{(1)}$.

\begin{tabular}{|c|c|c|c|c|}
\hline \multicolumn{5}{|c|}{ Legumes planta $^{-1}$} \\
\hline \multirow{2}{*}{ Método de pastejo } & \multicolumn{3}{|c|}{ Intensidade de pastejo } & \multirow[t]{2}{*}{ Média } \\
\hline & Moderada & Baixa & Sem pastejo & \\
\hline Contínuo & $43 \pm 6,9 b$ & $57 \pm 9,4$ a & $\ldots$ & $50 \pm 8,1 \mathrm{~A}$ \\
\hline Rotacionado & $35 \pm 8,7 \mathrm{a}$ & $44 \pm 13,1 \mathrm{a}$ & $\ldots$ & $40 \pm 10,9 \mathrm{~B}$ \\
\hline Média & $39 \pm 7,8 b$ & $51 \pm 11,2$ a & $\ldots$ & $\ldots$ \\
\hline Média & \multicolumn{2}{|c|}{$45 \pm 9,5$ a } & $28 \pm 11,1 b$ & $\cdots$ \\
\hline \multicolumn{5}{|l|}{ Peso de 1000 grãos } \\
\hline \multirow{2}{*}{ Método de pastejo } & \multicolumn{3}{|c|}{ Intensidade de pastejo } & \multirow[t]{2}{*}{ Média } \\
\hline & Moderada & Baixa & Sem pastejo & \\
\hline Contínuo & $134,4 \pm 10,6$ a & $141,6 \pm 11,8$ a & $\cdots$ & $138,0 \pm 11,2 \mathrm{~A}$ \\
\hline Rotacionado & $135,6 \pm 16,1 \mathrm{a}$ & $132,8 \pm 12,5 \mathrm{a}$ & $\ldots$ & $134,2 \pm 14,3 \mathrm{~A}$ \\
\hline Média & $135,0 \pm 13,3$ a & $137,2 \pm 12,1 \mathrm{a}$ & $\cdots$ & $\ldots$ \\
\hline Média & \multicolumn{2}{|c|}{$136,1 \pm 12,7$ a } & $112,5 \pm 13,0 b$ & $\ldots$ \\
\hline
\end{tabular}

Coeficiente de variação $(\mathrm{CV})=25,90 \%$ e 10,02\%, para legumes planta ${ }^{-1}$ e peso de 1000 grãos respectivamente.

(1) Médias com respectivo erro padrão, seguidas de letras minúsculas iguais na linha e maiúsculas iguais na coluna, não diferem significativamente pelo teste DMS a 5\% de significância.

Ciência Rural, v.38, n.3, mai-jun, 2008. 
Tabela 4 - Matéria seca de plantas daninhas ( $\mathrm{g} \mathrm{m}^{-2}$ ), na soja, nos métodos e nas intensidades de pastejo em sistema de integração lavourapecuária. Estação Experimental Agronômica - UFRGS, 2003/2004 ${ }^{(1)}$.

\begin{tabular}{|c|c|c|c|c|}
\hline \multirow{2}{*}{ Método de pastejo } & \multicolumn{3}{|c|}{ Intensidade de pastejo } & \multirow{2}{*}{ Média } \\
\hline & Moderada & Baixa & Sem pastejo & \\
\hline Contínuo & $6,68 \pm 0,99 \mathrm{a}$ & $2,26 \pm 0,36 b$ & $\ldots$ & $4,47 \pm 0,68 \mathrm{~A}$ \\
\hline Rotacionado & $7,71 \pm 0,89 \mathrm{a}$ & $2,11 \pm 0,38 b$ & $\ldots$ & $4,91 \pm 0,64 \mathrm{~A}$ \\
\hline Média & $7,20 \pm 0,94 \mathrm{a}$ & $2,19 \pm 0,37 b$ & $\cdots$ & $\cdots$ \\
\hline Média & \multicolumn{2}{|c|}{$4,70 \pm 0,66 \mathrm{a}$} & $2,68 \pm 0,48 b$ & $\ldots$ \\
\hline
\end{tabular}

Coeficiente de variação $(\mathrm{CV})=45,1 \%$.

(1) Médias com respectivo erro padrão, seguidas de letras minúsculas iguais na linha e maiúsculas iguais na coluna, não diferem significativamente pelo teste de DMS a 5\% de significância.

pastejo baixa e moderada, respectivamente, demonstrando controle mais efetivo devido a maior massa residual de azevém no tratamento com intensidade de pastejo baixa no momento da semeadura direta da soja.

\section{CONCLUSÕES}

A presença de pastejo por cordeiros em azevém, em sistema de integração lavoura-pecuária, favorece o rendimento de soja. A baixa intensidade de pastejo com cordeiros durante a estação fria propicia maior rendimento da soja, expressa pelo maior número de legumes por planta. O método de pastejo contínuo ou rotacionado com cordeiros, em pastagem de azevém, não são determinantes no rendimento da soja em sucessão.

\section{AGRADECIMENTOS}

À Empresa Agropecuária Cerro Coroado, pela concessão dos animais aos integrantes do Grupo de Pesquisa em Ecologia do Pastejo, à Coordenação de Aperfeiçoamento de Pessoal de Nível Superior (CAPES) e ao Conselho Nacional de Desenvolvimento Científico e Tecnológico (CNPq).

\section{REFERÊNCIAS}

ASSMANN, T.S. Rendimento de milho em áreas de integração lavoura-pecuária sob o sistema de plantio direto, em presença e ausência de trevo branco, pastejo e nitrogênio. 2001. 80f. Tese (Doutorado em Agronomia Produção Vegetal) Setor de Ciências Agrárias, Universidade Federal do Paraná, Curitiba -PR.

BERTOL, I. et al. Propriedades físicas do solo relacionadas a diferentes níveis de oferta de forragem de capim-elefante anão cv. Mott. Pesquisa Agropecuária Brasileira, v.35, n.5, p.1047-1054, 2000.

BICKI, T.J.; SIEMENS, J.C. Crop response to wheel traffic soil compaction. Transactions of the ASAE, v.34, n.3, p.909913, 1991.
CANARACHE, A. et al. Effect of induced compaction by wheel traffic on soil physical properties and yield of maize in Romania. Soil \& Tillage Research, v.4, n.2, p.199-213, 1984.

CARVALHO, P.C.F. et al. O estado da arte em integração lavoura e pecuária. In: CICLO DE PALESTRAS EM PRODUÇÃO E MANEJO DE BOVINOS, 10., 2005, Porto Alegre. Anais... Canoas: ULBRA, 2005. p.7-44.

CASSOL, L.C. Relações solo-planta-animal num sistema de integração lavoura-pecuária em semeadura direta com calcário na superfície. 2003. 143 f. Dissertação (Doutorado em Agronomia - Programa de Pós-graduação em Ciência do Solo) - Departamento de Solos, Universidade Federal do Rio Grande do Sul.

COMISSÃO DE FERTILIDADE DO SOLO-RS/SC. Recomendações de adubação e de calagem para os estados do Rio Grande do Sul e de Santa Catarina. 3.ed. Passo Fundo: SBCS - Núcleo Regional Sul, 1995. 224p.

EMBRAPA, Centro Nacional de Pesquisas de Solos. Sistema Brasileiro de Classificação de Solos. Brasília: Embrapa produção de informação. Rio de Janeiro: Embrapa solos, 1999. 412p.

FONTANELI, R.S. et al. Análise econômica de sistemas de produção de grãos com pastagem anuais de inverno, em sistema plantio direto. Pesquisa Agropecuária Brasileira, v.35, n.11, p.2129-2137, 2000.

FREITAS, T. M. S de. Dinâmica da produção de forragem, comportamento ingestivo e produção de ovelhas Ile de France em pastagem de azevém anual (Lolium multiflorum Lam.) em resposta a doses de nitrogênio. 2003. $123 \mathrm{f}$. Dissertação (Mestrado em zootecnia) - Programa de Pósgraduação em Zootecnia, Faculdade de Agronomia, Universidade Federal do Rio Grande do Sul.

MEDEIROS, R.B.; NABINGER, C. Rendimento de sementes e forragem de azevém anual em resposta a doses de nitrogênio e regimes de corte. Revista Brasileira de Sementes, v.23, n.2, p.245-254, 2001.

MORAES, A.; LUSTOSA, S.B.C. Efeito do animal sobre as características do solo e a produção da pastagem. In: SIMPÓSIO SOBRE AVALIAÇÃO DE PASTAGENS COM ANIMAIS, 1997, Maringá. Anais... Maringá: UEM, 1997. p.129-149. 
MOTT, G.O.; LUCAS, H.L. The design, conduct and interpretation of grazing trials on cultivated and improved pastures. In: INTERNATIONAL GRASSLAND CONGRESS, 6., 1952, State College. Proceedings... Pennsylvania: State College, 1952. p.1380-1385.

PONTES, L.S. et al. Fluxo de biomassa em pastagem de azevém anual (Lolium multiflorum Lam.) manejada em diferentes alturas. Revista Brasileira de Zootecnia, v.32, n.4, p.814-820, 2003.

PONTES, L. S, et al. Variáveis morfogênicas e estruturais de azevém anual (Lolium multiflorum Lam.) manejado em diferentes alturas. Revista Brasileira de Zootecnia, v.33, n.3, p.529-537, 2004.
SAS INSTITUTE. SAS/STAT user's guide: statistics. 5.ed. Version 8.02. Cary, NC, 2001. V.2. 943p.

THOMAS, A.L. et al. Rendimento de grãos de soja afetado pelo espaçamento entre linhas e fertilidade do solo. Ciência Rural, v.28, n.4, p.543-546, 1998.

TREIN, C.R. et al. Métodos de preparo do solo na cultura do milho e ressemeadura do trevo, na rotação aveia + trevo/milho após pastejo intenso. Revista Brasileira de Ciência do Solo, v.15, n.1, p.105-111, 1991.

VENTIMIGLIA, L.A. et al. Potencial de rendimento da soja em razão da disponibilidade de fósforo no solo e dos espaçamentos. Pesquisa Agropecuária Brasileira, v.34, n.2, p.195-199, 1999. 